\title{
ON INDEPENDENT DOMINATION IN PLANAR CUBIC GRAPHS
}

\author{
Gholamreza Abrishami $^{a}$ \\ Michael A. Henning ${ }^{b, 1}$ \\ AND \\ FREYDOON RAHBARNiA $^{a, 2}$ \\ a Department of Applied Mathematics \\ Ferdowsi University of Mashhad \\ P.O. Box 1159, Mashhad 91775, Iran \\ ${ }^{b}$ Department of Pure and Applied Mathematics \\ University of Johannesburg \\ Auckland Park, 2006 South Africa \\ e-mail: gh.abrishamimoghadam@mail.um.ac.ir \\ mahenning@uj.ac.za \\ rahbarnia@um.ac.ir
}

\begin{abstract}
A set $S$ of vertices in a graph $G$ is an independent dominating set of $G$ if $S$ is an independent set and every vertex not in $S$ is adjacent to a vertex in $S$. The independent domination number, $i(G)$, of $G$ is the minimum cardinality of an independent dominating set. Goddard and Henning [Discrete Math. 313 (2013) 839-854] posed the conjecture that if $G \notin\left\{K_{3,3}, C_{5} \square K_{2}\right\}$ is a connected, cubic graph on $n$ vertices, then $i(G) \leq \frac{3}{8} n$, where $C_{5} \square K_{2}$ is the 5 -prism. As an application of known result, we observe that this conjecture is true when $G$ is 2-connected and planar, and we provide an infinite family of such graphs that achieve the bound. We conjecture that if $G$ is a bipartite, planar, cubic graph of order $n$, then $i(G) \leq \frac{1}{3} n$, and we provide an infinite family of such graphs that achieve this bound.
\end{abstract}

Keywords: independent domination number, domination number, cubic graphs.

2010 Mathematics Subject Classification: 05C69, 05C10.

\footnotetext{
${ }^{1}$ Research supported in part by the South African National Research Foundation and the University of Johannesburg.

${ }^{2}$ Corresponding author.
} 


\section{REFERENCES}

[1] C. Barefoot, F. Harary and K.F. Jones, What is the difference between the domination and independent domination numbers of a cubic graph?, Graphs Combin. 7 (1991) 205-208. doi:10.1007/BF01788145

[2] P. Dorbec, M.A. Henning, M. Montassier and J. Southey, Independent domination in cubic graphs, J. Graph Theory 80 (2015) 329-349. doi:10.1002/jgt.21855

[3] W. Goddard and M.A. Henning, Independent domination in graphs: A survey and recent results, Discrete Math. 313 (2013) 839-854. doi:10.1016/j.disc.2012.11.031

[4] W. Goddard, M. A. Henning, J. Lyle and J. Southey, On the independent domination number of regular graphs, Ann. Comb. 16 (2012) 719-732. doi:10.1007/s00026-012-0155-4

[5] W. Goddard and J. Lyle, Independent dominating sets in triangle-free graphs, J. Comb. Optim. 23 (2012) 9-20. doi:10.1007/s10878-010-9336-4

[6] T.W. Haynes, S.T. Hedetniemi and P.J. Slater, Fundamentals of Domination in Graphs (Marcel Dekker, Inc. New York, 1998).

[7] M.A. Henning, C. Löwenstein and D. Rautenbach, Independent domination in subcubic bipartite graphs of girth at least six, Discrete Appl. Math. 162 (2014) 399-403. doi:10.1016/j.dam.2013.08.035

[8] A.V. Kostochka, The independent domination number of a cubic 3-connected graph can be much larger than its domination number, Graphs Combin. 9 (1993) 235-237. doi:10.1007/BF02988312

[9] P.C.B. Lam, W.C. Shiu and L. Sun, On independent domination number of regular graphs, Discrete Math. 202 (1999) 135-144. doi:10.1016/S0012-365X(98)00350-1

[10] J. Lyle, A note on independent sets in graphs with large minimum degree and small cliques, Electron. J. Combin. 21 (2014) \#P2.38.

[11] O. Ore, Theory of graphs, Amer. Math. Soc. Transl. 38 (1962) 206-212. doi:10.1090/coll/038

[12] J. Southey and M.A. Henning, Domination versus independent domination in cubic graphs, Discrete Math. 313 (2013) 1212-1220. doi:10.1016/j.disc.2012.01.003

[13] T. Zhu and B. Wu, Domination of maximal $K_{4}$-minor free graphs and maximal $K_{2,3}$-minor free graphs, and disproofs of two conjectures on planar graphs, Discrete Appl. Math. 194 (2015) 147-153.

doi:10.1016/j.dam.2015.05.029

Received 2 August 2017

Revised 1 December 2017

Accepted 1 December 2017 